\title{
Who Needs The Teacher?
}

Alexander Vengerov, Ramapo College of NJ, USA

\begin{abstract}
The purpose of the present paper is to establish, define, and develop a high-level architecture for Systems of Learning from the viewpoint of interdependencies between the processes of selforganization and collective intelligence. The role of the Teacher/Manager therefore changes to the collective learning and intelligence taking the central stage. The paper also develops and justifies several views within the stated viewpoint serving better understanding of the relationship between these processes and offering a common ground for the discussion and development of various implementation scenarios.
\end{abstract}

Keywords: Collective Learning; Collective Intelligence; Self-Organization; Architecture; Architectural Pattern

\section{INTRODUCTION}

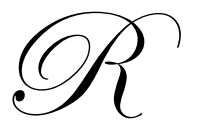

apid growth of interwoven collective behavior has led to an explosion in social networking and Web 2.0 operations. Today's social networks already count close to one billion members worldwide. Facebook, the most popular OSN, has more than 500 million active users [1], and has surpassed Google as the most visited site on the Internet [2]. Increasingly, Facebook and Twitter are replacing email and search engines as users' primary interfaces to the Internet [3]. William Baker, a professor of marketing at San Diego State University, surveyed 1,600 executives and found that firms that rely heavily on external social networks scored 24 percent higher on a measure of radical innovation than companies that don't [4].

Low effectiveness of information transfer (lecturing) model of learning as well as its low scalability and low efficiency were discussed above. This, in the face of the growth of communication/collaboration (Web 2.0 style technologies) and intensity of social interactivity, creates a bottleneck not only in educational services in academia but in embedded forms of learning in organizations and other socio-economic processes. The choice of learning organization with one hub and students as spokes on the receiving ends just doesn't support the necessary effectiveness, efficiency and scalability according to the new developing situation of growing needs in more adequate forms of learning in various learning environments. The growing importance of such non-functional requirements [45] as agility, autonomy, complexity, cost, distribution, evolvability, flexibility, modifiability, openness, and adaptivity lead to the need in the change of the way systems of learning are being designed and implemented.

The purpose of the present paper is to establish, define, and develop a high-level architecture for Systems of Learning (SoL) from the viewpoint of interdependencies between the processes of self-organization, collective learning, and collective intelligence. The paper also develops and justifies several views within the stated viewpoint serving better understanding of the relationship between these two processes and offering a common ground for the discussion and development of various implementation scenarios. It is an attempt to develop a theoretical framework for better understanding of the phenomenon of distributed collective learning and corresponding effects of collective intelligence in a sufficiently general architectural framework allowing for SoL with large self-organization component.

The importance and possibility of developing architectural foundation for such systems is based on the rapid growth of communication technologies and their use in a vast number of activities that previously were under centralized control. As a result, distributed development and administration of complex socio-technological systems is becoming a major form of coping with growing complexity and speed of change. Distributed operations in changing environment require solid foundation of continuous distributed learning without spending all employee 
time in attending courses and learning boot camps (efficiency of learning). At the same time there should be lower teaching without hiring an army of instructors, seeking multiple classroom spaces, equipment, bureaucracy, etc. (efficiency of teaching).

The rest of this paper is organized as following: section 1 presents the findings of related research literature; section 2 discusses the proposed CL/CI architectural pattern; and section 3 discusses methods of Collective Intelligence (CI) implementation in order to support Collective Learning (CL).

\section{PART 1: MANAGEMENT AND SELF-ORGANIZATION OF CI AND CL}

The concepts of collective learning and collective intelligence form strongly interdependent and somewhat overlapping domains. Yet, study of these processes took largely separate paths. The concept of Collective Intelligence has been studied for quite a while in works dealing with various biological mechanisms like ant and bee swarm intelligence [5,6], as well as in human [7,8,9] and AI systems. CI among humans can be distinguished as harvested (and then applied in designer efforts) or self-emerging to its active role [10,11].

Lately the focus started shifting to more complex configurations of CI that include human, automated, and environmental factors. The Center for Collective Intelligence at MIT states that their focus is on understanding of "How can people and computers be connected so that-collectively-they act more intelligently than any individuals, groups, or computers have ever done before?" [12]

Collective learning is seen as "a social process that produces intellectual synergy of many minds coming together to bear on a problem, and the social stimulation of mutual engagement in a common endeavor. This mutual exploration, meaning-making, and feedback often lead to better understanding." [13]. The foundation of CL can be traced to constructivist and social cognitivist learning theories [14]. Other learning theories laying ground for the idea of collective learning include concepts of systems vision of learning situation [15, 16], distributed cognition $[17,18,19]$, gestalt theory [20,21], problem-based learning [22,23], cognitive apprenticeship [24, 25], and situated learning [26, 27]. Each of these learning theories focuses on the social aspect of learning and knowledge building. Each theory recognizes that learning and knowledge building involve inter-personal activities including conversation, argument, and negotiation [28]. In this sense the role of Learning Environments is changing from passive environmental boundaries to an active cooperative component that has to be a part of the description of the key architectural parts of any CL/CI system.

From the angle of analysis taken in this paper we would distinguish two contrasting paradigms of CL: control and self-organization. Within control approach the efficiency of learning is based on well designed and tightly managed learning processes. In self-organization approach the creative power of the collective as well as additional components of respective learning environments (such as intelligent agents, means of communication and information processing, smart objects, etc.) are the key.

\section{Controlled Collective Learning and Intelligence}

One of the most widespread attitudes to learning in organizational setting is in treating it as a controlled process aiming at delivery of either tangible values for the organization or, at least, some demonstration of completed "information transfer" as knowledge "acquisition." Collective learning has been seen as ability of a collective in its organizational environment to implement an adequate organizational change [33].

Planning learning goals and deriving the structure of the heavily controlled learning processes can be supported by a number of techniques and modeling languages allowing for their design [29, 30, 31]. The traditional goal of the CL design was in having sufficiently scripted and managed details of the learning process in its learning environment (including goals, resources, technologies, the exact nature of prescribed collaborative tasks, roles of collaborators, motivations, learning settings, conditions, time periods, etc.) [32].

A number of forms of CL like Group Decision Support, Communities of Practice (CoP), collaborative learning (usually in teams), open source and agile project development, mediated/controlled discussions, and some 
other similar forms share the existence of an "entity in charge" (appointed, elected, or evolved and accepted as such) with varying degrees of management interventions. In the world of automated means supporting such approach to learning we can name supervised learning methods [34].

\section{Self-organization of Collective Learning and Intelligence}

An alternative focus on emergent and self-organization properties of CL/CI was taken by another group of researches. Already Wheeler [35] saw a collective as a "superorganism" with intelligence of its own, which was "a complex, definitely coordinated and therefore individualized system of activities, which are primarily directed to obtaining and assimilating substances from an environment, to producing other similar systems, known as offspring, and to protecting the system itself and usually also its offspring from disturbances emanating from the environment" (p. 308). An important aspect of such collective form was the existence of new features that no parts had separately. "The unique qualitative character of organic wholes is due to the peculiar non-additive relations or interactions among their parts. In other words, the whole is not merely a sum, or resultant, but also an emergent novelty, or creative synthesis." [36]

The roots of collective learning in human collectives are stemming from Vygotsky's work on the role of social interactions in producing results that can normally be attributed only to knowledge acquisition (but without any entity actually acquiring knowledge). Thus Vygotsky [8] notes that: "Every function in the child's cultural development appears twice: first, on the social level, and later, on the individual level; first, between people (interpsychological) and then inside the child (intrapsychological). This applies equally to voluntary attention, to logical memory, and to the formation of concepts. All the higher functions originate as actual relationships between individuals." (p57).

Later in the framework of studies of complexity [37] it was discovered that these emergent features happen only when the level of mutual sensitivity of elements/actors in the collective exceeds certain minimum. One of the fields strongly affected by the increased scale of interactivity supported by a new wave of ICT capabilities was learning. Understanding of educational essence started shifting away from discredited instructional paradigms to more socially situated forms of learning $[38,39]$.

Despite the increasing appearance of more autonomous and self-organized ways of SoL, some researchers of CL think that the efforts should be focused not as much on the amazement in watching self-organization that has no guarantee of evolving in the desirable direction as to do more control over the actual settings of the CL situation [40]. They point out that the very nature of learning via distribution and interaction cannot be seen as a methodology since there is no guarantee that the benefits will outweigh the shortcomings. High sensitivity of CI settings to the variety of conditions [40] shows lack of guaranteed methods of "doing collaboration right."

Important to understand that "mixing" controlled and self-organized collaboration is not easy. A defining characteristic of social [collective] creativity is that it transcends individual creativity and thus requires some form of organization; but elements of organization can and frequently do stifle creativity [41]. This shows the need in seeking more fundamental patterns, architectures, and concepts allowing further improvements in using benefits of both approaches (control and self-organization) while trying to avoid their shortcomings by creating proper synergies in learning designs and architectures as well as foundation for a collective discussion of this phenomena. The rest of the paper focuses on the development of architectural views highlighting the important structural and behavioral components of CL/CI that can serve as a common ground for such collective discussion, analysis, and implementation efforts.

\section{P2: CL/CI ARCHITECTURE}

By fusing CI and CL we want to emphasize their essence as inseparable and dynamic counterparts. CL in such context is seen as a process of learning using mixed individual and collective resources where collective efforts, exchange, and collaboration are used on all or only some stages and segments of learning. For example, we can see influence of collective learning enhancements on individual learning (IL) in such areas as perception, experience, emotional response, reactive actions, methods and approaches to learning, rationalization and 
internalization that result in changes in the state of Individual Intelligence (II) as well as CI.

This makes CI (in totality of its forms) a process of emergence of a Teacher/Course Manager role. The link between collective forms of learning and CI allows switching the discussion from traditional managed forms of learning/education with the role of a teacher as lecturer/manager or, in best cases, a helper in the background, to elimination of a traditional human Teacher role at all. At the same time the instructor keeps playing an important role as a "gardener" of the growth of individual and collective intelligence, supporting effectiveness and efficiency of individual and collective learning in their interwoven forms benefiting from and contributing to individual and collective intelligence.

Additionally, we want to note that the growing role and use of CL and CI, that are not well defined and understood, leads to the need of the formation of the common conceptual ground for discussion and design of such systems. One of the methods allowing to decrease the multitude of problems in developing and supporting various forms of collective learning, especially with attempts of harnessing CI, is in the development of architectural views that might serve as organizing vehicles for common discussions and management/maintenance efforts. The paper proposes two high-level views on CL/CI architecture covering the composition and interaction of the main parts along with methodological view on how to handle such design.

The architectural pattern (AP) proposed in this section realizes a view offering a generalized platform for discussions, analysis, and the design of SoL with planned large self-organization component and the need to alleviate scalability problems. Architecture is understood as all that which is essential or unifying about a system; the set of properties of a system which determine the system's structure and behavior. It can address such growing concerns in developing SoL as: agility, autonomy, complexity, cost, distribution, evolvability, flexibility, modifiability, openness, state change and adaptivity. This is why the latest revision of IEEE 1471 standard places focuses on the dynamic versus old static features of architectural design. It emphasizes the importance of the architectural design in "expression of the systems and its evolution, in expression of persistent characteristics and supporting principles of a system to guide acceptable change" [42]. According to the conceptual framework established by IEEE 1471 [43] an architectural description is organized into one or more constituents called (architectural) views. Each view addresses one or more of the concerns of the system stakeholders. A view is a partial expression of a system's architecture with respect to a particular viewpoint. An architectural description (AD) selects one or more viewpoints for use. The selection of viewpoints is typically based on consideration of the stakeholders to whom the $\mathrm{AD}$ is addressed and their concerns.

The viewpoint presented here offers the vision of SoL as mainly a self-organized process with the need of balancing delicate holistic methods (therapy) supporting healthy self-organization processes with the control approach that is forcefully changing internal architectural features (surgery) and often leading to the need in healing and re-developing vital harmonies upset by such intervention. Such self-organization viewpoint on SoL is further detailed in two views: CL/CI Architecture (presented in this section) and CL/CI Implementation Model (presented in the next section).

SoL with large self-organization share of processes face similar problems in CL organization and are largely interdependent with CI. Such commonality creates the necessity and possibility of the development of architectural views that can function as a reusable pattern. The paper offers a viewpoint with several CL/CI architectural views designed to provide a common foundation for the analysis of the described type of Sol. The first view (see fig.1) focuses on high-level dynamic convolution of efforts of Individual Learners (IL) in Collective Learning environment and the role of Collective Intelligence (CI) in their respective contexts and environments. The second view will cover the implementation methods of CI incorporation.

An example of encountered problems addressed by CL/CI can be seen in courses with dynamic content and rapidly evolving domains of knowledge (like Electronic Business and Web Development). The courses are a part of Web Development Certificate program and theoretically can be taken by any person, not just students with sufficient prerequisite knowledge and skills. Attempts of using social networks for enhanced learning from each other were not very successful since in the controlled version students loose energy of self-organization and creativity, leading to the need of more control, and so on. In addition, using closely managed groups just doubles the instructor's work 
load since in addition to controlling individuals there is a need in managing groups. This latter idea is being developed as CL/CI architecture and discussed in the current paper. On the other hand, if peer interaction in social network environment is left uncontrolled, the effectiveness (for the intended learning goals) becomes poor with a lot of low value chit-chat. As a result, there was a perception of the need either in getting more people involved in teaching growing size of classes in their increased interactive dynamism (creating efficiency as well of effectiveness problems) or summon help from CI incorporation.

The use of the described below AP is based on the situation when the designers/stakeholders of the SoL development/use/maintenance made a conscious decision of harnessing the combination of forces of selforganization and CI for the benefit of enhancing the effectiveness and efficiency of the learning process. At the same time, they want to preserve some level of control over the way such self-organization works since, as it is known from the Dynamical Systems theory, not all self-organized processes can result in viable, and useful dynamics (e.g. crowd madness, population extinction, etc.). Another assumption is that means and skills of implementing various forms of AI and advanced IT processes supporting CI are inexpensive and readily available for the project. Also, learners have to have broadband Internet connection and ability to dedicate enough time for intensive interactive regimes. They also have to be motivated in the success and sufficiently manageable, when necessary.

Structure and Interactions of CL/CI pattern focus on the following key components determining the evolvability and adequacy of the whole system:

a.i.1. Individual learners (IL) in the presented AP can be seen in one of two roles (or both at the same time):

a.i.1.a. as beneficiaries of the learning process (learned something)

a.i.1.b. as providers of their share of CI as well as of other benefits for the whole SoL or its parts (like project workability, process adequacy, unit viability, etc.).

a.i.2. Individual Intelligence (II) and Individual Learning (IL) efforts represent individual learning behavior, actions, choices and the accrued knowledge as a result of learning which can further affect the way individuals choose to learn.

a.i.3. Collective Intelligence (CI) and Collective Learning (CL) are counterparts of II/IL but seen as collective in the context of collective efforts.

a.i.4. Information and Communication Technologies (ICT) are supposed to support all technological and interactivity needs of other architectural components thus allowing for the new CL/CI teaching paradigm to be implemented

a.i.5. Advanced Intelligence Technologies (AIT) block deals with special methods, structures, and processes supporting the discovery, formation, and use of the synergy between individual and collective intelligences in their learning efforts.

a.i.6. Learning Environment (LE) includes all other environmental components that are not current members of ATC.

In addition we must say that the problem of such blending stems from the need in balancing coexistence and competition of two often conflicting efforts of course management: self-organization and control. Compared to the old instructional design paradigm the CL/CI architecture uses two components as sources of external interventions: Management Mechanisms (MM) with adaptive means allowing to instill some "rules of the game" but also modify them as necessary [see my MSML paper] and Instructional Design (ID) or learning design as a more static intervention than the use of MM. Note that both methods should consider the constraints of each other (link M10). 


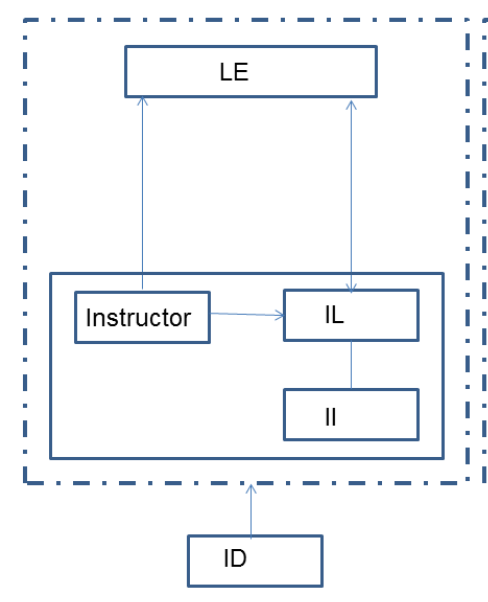

Fig. 1 (a)

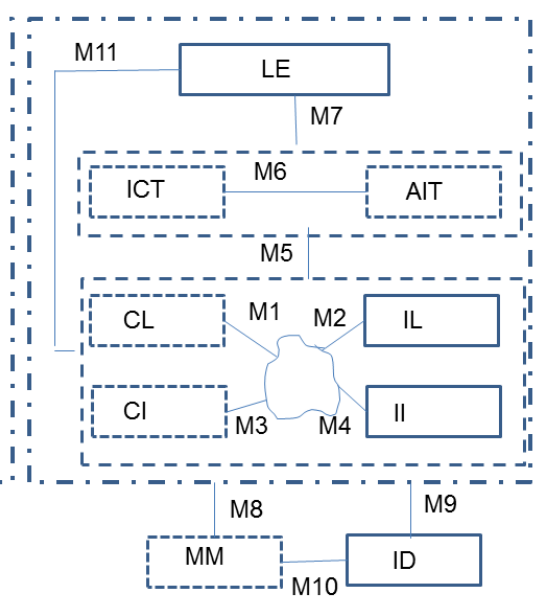

Fig. 1 (b)

Comparison of the Traditional Fig. 1(a) and CL/CI Architecture Fig. 1(b)

The key to the understanding and properly using the AP is in its specific features compared to usual systems (and especially software) architectures and nature of interactions among its components. Thus the $\underline{\mathbf{C L} / \mathbf{C I}}$ pattern (see fig.1b) has specific properties compared to the usual entity-connectors architectural views (fig 1a). Some components on CL/CI pattern are shown as dashed shapes emphasizing the fuzzy, evolving nature of the underlying concepts with dynamic permeable boundaries. The main sections of the pattern can be divided into two subsections. One forms the most active and dynamically evolving area of contextual relationships periodically forming more stable patterns optimizing their mutual harmonization processes. This part includes CL, CI, IL, and II. Another part includes the relationships with advanced intelligence (AIT), communication and information technologies (ICT) and Learning Environment (LE), which is understood broader than just an LMS. It is seen more along a constructivist concept including at least four components: an enabling context (or enabling environment - our comment), resources, a set of tools, and scaffolds [46].

This division and its architectural consequences are important for the understanding of the CL/CI pattern. In traditional architecture (fig. 1a) Instructor and Individual Learner have clearly defined roles and hierarchical interactions. Learning Environment in this case is just a management resource. Separation into Environment plus Technology and Pattern-Context dynamics of the main CL/CI operations (as shown on fig. 1b) was explored in other architectural models [47]. This division is not set in stone and some pattern-context complexes can be moved to Environment or considered to be parts of Technological Support as not playing active role in the evolving of the key learning dynamics, while some environmental and technological components can later become a part of the patterncontext area. This area is usually the heart of the dynamism of evolving and adaptive processes in complex systems. For example, adaptive feedback from the training program to an individual student during topic learning or testing immediately affects the mode of learning (like an advice to re-read a chapter, do a specific simpler task before starting the difficult one, seek help from the peers or instructors, etc.). On the other hand such events like the change of a specific email provider with slightly different email features do not affect the dynamism of learning while maintaining certain constraints on emailing activity and, therefore, can be seen as a part of the LE/Technology complex.

Such evolving and mutually applicable nature of the used concepts creates a recurrent architectural pattern where every block can be analyzed through the properties of all other blocks. For instance, the MM block representing Management Mechanisms interventions into the self-organization of CL/CI dynamics in their interaction with LE/Technology can by itself be seen on a lower level as a system utilizing the methods of selforganization and CL/CI dynamics. Thus there are many examples when dynamically created virtual groups of 
learners started developing sufficiently stable traits and norms that were fed back into the collective as external controlling necessity to the current creativity and behavioral variance. Such recurrent nature of CL/CI view under environmental and management pressures reflects a general reoccurring situation that can be seen as a pattern of the new reality and used in analysis and discussions of the components, relations, and their dynamics. Other details of this view include relationships among the shown blocks.

IL use self-organization and evolving during the learning process leading to the continuous change of their state of skills, knowledge, and orientation. I want to emphasize that these are not typical objects in architectural drafts but rather pointers at continuously evolving and changing processes/states. Thus the new state of the collective of individual learners leads to the new state of CI, which due to the M1-M4 feedbacks affects the process of IL evolution or changes the state of an IL directly (as in planning learning activities and assigning individual roles, steps, and due dates). IL can also affect LE and Technology Block via M11 and M7. A number of learning theories discussed in part 1 emphasize the relationship with environment in IL state formation where LE is not seen as a controlling/management system. One of the examples is project-based learning where the test of the project workability in LE creates a teaching feedback, leading to the correction of understanding of the situation and possibly leading to additional chains of learning activities. Another example of a non-management influence of LE is in learning programming languages. There students, after reading the lesson, might feel that they understand it, but the real test comes during developing the workable program, which often requires rethinking of the learned concepts and acquiring additional knowledge/skills to be adequate to the emerging design/debugging situation.

CL and CI, strongly depending on IL/II changes, often demonstrate properties known as "mind of society" [8]. Important to note that the formation of CI as well as its impact on IL can be potential or actual depending on the ability to recognize and accumulate CI-related phenomena from IL activities and II-states. Such functions could be a result of human actions in the main learning blocks, instructor's actions, or done automatically by using the advanced technology of ICT/AIT mutually enhancing each other via M6 interdependence.

Instructional Design (ID) in the proposed architecture works differently than in traditional learning architectures (fig. 1a) where it provides a one-way controlling influence on SoL, which is largely static during the learning process. The new approach creates a mutually corrective connection (M10) between two complementary types of influences of the Management Mechanisms (MM) and Instructional Design (ID). The dynamic combination of these two actors creates the possibility of invasive, control-type intervention (M9) and a more holistic-style minimum invasive influence (M8) that allows the system to form a new pattern of behavior out of the modified context (of M1-M4 interactions) on its own, versus directly changing the patterns of individual and collective learning states and behaviors.

If in traditional instructivist approach the role of Instructional Design with its control style loops of analysis, design, development, implementation, and evaluation is considered to be still dominant for the learning outcomes (see fig 1a), the further growth of the dynamism of learning processes have led to a more flexible and adaptive concept of Learning Design, which according to the IMS Learning Design specification [48] supports the use of a wide range of teaching methods in online learning by using a generic language. Our architectural pattern reflects a further development of such language generality by extending it to the level when self-organization substitutes the control function of the instructor/course manager. Accommodation of this new reality forms the Pattern-Context dynamics of the offered AP. The main idea here is to dramatically diminish the role of preliminary instructional/learning "design" with emphasis on creative evolution of the learning processes in a non-didactic selforganized manner.

\section{P3: INCORPORATION OF COLLECTIVE INTELLIGENCE} including:

The second view (see Fig. 2) is more methodological and describes a detailed process of CI harnessing,

A. Determining methodological approach (richness, breadth, integration, focus)

B. Detecting and eliciting CI-relevant data in accordance with step A

C. Processing CI-relevant data obtained on step B into value-based intelligence

D. Implementing of the obtained CI in order to provide the value/utility determined on step C 


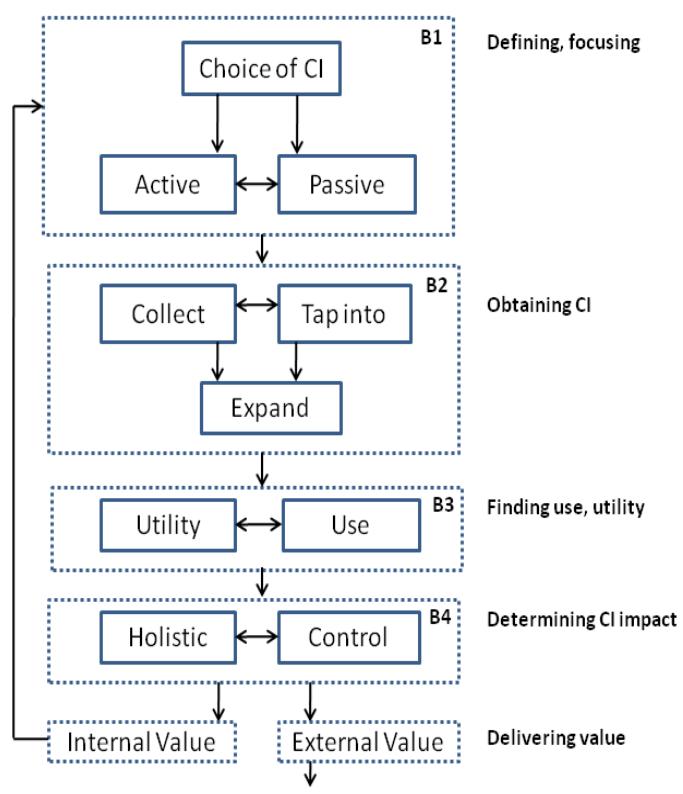

Fig 2. CI Incorporation Model

\section{Determining methodological approach (block B1)}

As we mentioned in the Introduction, the concepts of intelligence and collective intelligence are very fuzzy, poorly defined, and dynamically changing with new uses and views on the phenomenon. Although there can be many classifications and decisions to "go after" some of CI types, one of the most important decisions is in distinguishing two possible means of obtaining CI:

- $\quad$ conscious deliberate activity of individual actors (learners in our case) in the process of creation and using $\mathrm{CI}$;

- $\quad$ collection of passive but useful data accompanying collective behavior and discovered via some kind of mining of the broad behavioral characteristics of the collective (see block B1 on fig.2).

Each of these two methods (active and passive CI creation) can be further subdivided into more detailed categories that should be further discussed, understood, and made clear as goals, focus, and degree of richness and breadth of the CI concept that will be distinguished and pursued. The processes belonging to B1 are iterative since the consensus of what to understand and pursue as CI changes with CI embodiment in systems functionality. This requires periodic reassessment and even redesign of the approach to CI with new technologies unleashing new types of connectivity and CI manifestations. New utility forms of CI are adapting to these changes and continuously evolving making it a dynamic process in principle.

\section{Detecting and eliciting CI-relevant data (block B2)}

After the concept and the scope of CI that will be harnessed are determined in B1, it becomes a guiding part of the architecture for the block B2 dealing with the way it can be detected, collected, or redirected. The two big methodological types making CI available are seen in distinguishing context-sensitive (grounded) and context-free (movable) intelligence. If the second type has just to be detected, filtered, and collected (like student grades, statistics of viewing task pages and wiki pages, etc.), the first type cannot be separated from its context and can only be channeled as an additional feed into some other process. Examples of such types of context-sensitive intelligence 
can include the process of reviewing other students' work, replying to blog posts, etc. Each of them has value not as particular information that was provided by individuals but as existence of the corrective feedback. Such feedback might be valuable and either enhanced with additional features or channeled into other processes. For example, grading can be required in addition to the reviewing feedback thus creating a collective measure of student's performance. Discovered relevant Internet sources in addition to the regular process of updating a collective repository, might be directed to some kind of automated or manual classifier that will create a topical structure of the domain with ontologies or tag clouds helping the development of the Semantic Network. The lower level of decision-making in B2 design is the choice of specific methods and technologies allowing to detect and collect/direct CI that is chosen for further processing.

\section{Finding use/utility of obtained data access to CI ((block B3)}

Block B3 deals with selecting the valuable patterns/contexts from the ones detected and made available in B2. For example access to personal profiles doesn't mean intelligence by itself. There is a need to figure out what might be valuable for a particular system (like a particular SoL) and how to convert access to potentially useful sources of CI into the valuable and usable CI. A number of techniques can be applied here like clusterization, case building for CBR, classification, meaning extraction, etc.

\section{Determining CI impact (block B4)}

This is a very delicate step because in many systems (and SoL in particular) we are dealing with large areas of self-organization. Speaking of differentiation of CI impacts according to the CL/CI architecture discussed in the previous section, we can distinguish two basic approaches. The approaches can be called "surgery" and "therapy" differing in the way the target system for the planned value addition from CI performance is being treated. We can see two different roles of CI dispatcher in the controlling role: directed to some external stakeholder system or the system creating $\mathrm{CI}$ itself. In this case more bold intervention might take place up to dramatic re-engineering of the way the target system is functioning. This requires taking all responsibility for such changes as well as for the necessary re-harmonization (healing) of the existing preferences and broken harmonies.

An alternative way is in using use gentle holistic approach aiming at restoration of the health of selforganization itself. It is more akin to therapy (holistic medicine style) where the interventions into the target system, delivering benefits of $\mathrm{CI}$, are made in a way correcting and enhancing own systems self-organization and selfharmonization processes. The targets of these methods can include both directions of delivering benefits/utility: for the external environment (External Value on fig. 2) and/or for the system itself (Internal Value).

\section{Important features of the Methodological View}

All blocks on fig. 2 have iterative impacts on each other, therefore it is not a traditional waterfall model of systems design. Thus the discovery of the valuable types of intelligence or finding effective methods of increasing its value can affect the approach in B1 to what is the focus of CI/CL interactive enhancement. Another important aspect is in the need of dynamic balancing the blend of different methods and/or directions of their application. Such dynamic evolving character of the main processes underscores the importance of adequate dynamic self-controlling processes versus using predesigned architectural blueprints.

\section{CONCLUSION: CONSEQUENCES}

Presented CL/CI pattern and accompanying architectural views determine and clarify the main activities and interactions in the process of spiral enhancement of experience and knowledge of individual learners obtained in complex interactions with their collective intelligence. This allows for common discussion and development of architectures of SoL intending to use large self-organization and autonomous components. The presented viewpoint explores the interaction between individual learning and collective intelligence largely substituting the typical instructor. It doesn't mean that we try to simulate the instructor since the goal is in providing the necessary support and guidance allowing for effective and efficient progression of learning activities. This viewpoint was explored in two views analyzing the structure and emergence of such self-organized learning. 


\section{AUTHOR INFORMATION}

Dr Alexander Vengerov is an associate professor of Information Systems at Ramapo College of NJ. He chaired an All-College Teaching and Learning with Technology Committee, and is the president of Syslearn, Inc. developing learning systems and intelligent learning processes, as well as consulting in the area of business and academic organizational learning. E-mail: abvenger@ramapo.edu

\section{REFERENCES}

[1] Sorkin, A. R. Facebook tops 500 million users. New York Times Blog, June 2010

[2] Yarow, J. Facebook was more popular in the U.S. than Google last week. BusinessInsider.com, March 2010

[3] Gannes, L. When social replaces search, what can you do to monetize? GigaOM, March 2010

[4] Jake Swearingen (September, 2008). Social Networking for Business. Retrieved December 22, 2010 from http://www.bnet.com/article/social-networking-for-business/219914

[5] Dorigo, M., Maniezzo, V. and Colorni, A. 1996. Ant system: optimization by a colony of cooperating agents. IEEE Transactions on Systems, Man, and Cybernetics, Part B. 26, 1, 29-41;

[6] Pham, D. T., Ghanbarzadeh, A., Koc, E., Otri, S., Rahim, S. and Zaidi, M. 2006. The Bees Algorithm - A Novel Tool for Complex Optimisation Problems. Proceedings of IPROMS 2006 Conference

[7] Weschsler, D. 1971. Concept of collective intelligence. American Psychologist. 26, 10, 904-907.,

[8] Vygotsky, L.S. (1978). Mind in Society. Cambridge, MA: Harvard University Press.

[9] Howard Bloom, Global Brain: The Evolution of Mass Mind from the Big Bang to the 21st Century, 2000

[10] Brown, Philip; Lauder, Hugh (2000). "Collective intelligence". In S. Baron, J. Field \& T Schuller. Social Capital: Critical Perspectives. New York: Oxford University Press.

[11] Weiss, A. (2005). The Power of Collective Intelligence. Collective Intelligence, pp. 19-23

[12] Center for Collective Intelligence. MIT. http://cci.mit.edu/

[13] Golub, J. (Ed). Focus on Collaborative Learning. Urbana, IL: National Council of Teachers of English, 1988.

[14] Resta, P. \& Laferrière, T. (2007). Technology in Support of Collaborative Learning. Educational Psychology Review, 19, 65-83. DOI 10.1007/s10648-007-9042-7

[15] Peter Senge, Art Kleimer, Charlotte Roberts, and Bryan Smith (1994). The Fifth Discipline Fieldbook: Strategies and Tools to Build a Learning Organization. Doubleday, New York

[16] Dick, Walter, Lou Carey, and James O. Carey (2005) [1978]. The Systematic Design of Instruction (6th ed.). Allyn \& Bacon. pp. 1-12.)

[17] Perry, M. (2003). Distributed Cognition. HCI Models, Theories, and Frameworks: Toward an Interdisciplinary Science 193-223.

[18] Dror, I.E. \& Harnad, S. (eds.) (2008). Cognition Distributed: How Cognitive Technology Extends Our Minds.

[19] John Benjamins, Amsterdam. Gureckis, T.M. and Goldstone. R.L. (2006). Thinking in Groups. Pragmatics and Cognition, 14 (2), 293-311.

[20] Wertheimer, M. (1959). Productive Thinking (Enlarged Ed.). New York: Harper \& Row.

[21] Gibson, J.J. (1979). The Ecological Approach to Visual Perception. Boston: Houghton Mifflin.

[22] Barr RD and Tagg J, (1995): From teaching to learning - a new paradigm for undergraduate education. Change, Nov/Dec.:13-25

[23] Loyens, S., Magda, J., \& Rikers, R. (2008). Self-Directed Learning. Problem-Based Learning and its Relationships with Self-Regulated Learning. Educational Psychology Review, 20(4), 411-427.

[24] Aziz Ghefaili. (2003). Cognitive Apprenticeship, Technology, and the Contextualization of Learning Environments. Journal of Educational Computing, Design\& Online Learning, Vol. 4, Fall, 2003.

[25] Bandura, A. (1997). Social Learning Theory. Englewood Cliffs, NJ: Prentice-Hall.

[26] Brown, J.S.; Collins, A.; and Duguid, P. (1989). Situated Cognition and the Culture of Learning. Educational Researcher 18, no. 1 (January-February): 32-41.

[27] Lave, J. Cognition in Practice. New York: Cambridge, 1988.

[28] Resta, P. \& Laferrière, T. (2007). Technology in Support of Collaborative Learning. Educational Psychology Review, 19, 65-83. 
[29] Kueng P., Kawalek P. Goal-Based Business Process Models: Creation and Evaluation. Business Process Management Journal, 1997, Vol. 3:1, pp.17-38..

[30] VanLamsweerde, A. "Goal-Oriented Requirements Engineering: A Guided Tour" Proc of the 5th IEEE Int. Symp. on Requirements Engineering, pp:249-262, 2001

[31] Nurcan, S. and Rolland, C. "A Multi-Method for Defining the Organizational Change" Information and Software Technology Journal 45 (2003) 61-82.

[32] Kobbe, L., Weinberger, A., Dillenbourg, P., Harrer, A., Hämäläinen, R., Häkkinen, P. \& Fischer, F. (2007) Specifying computer-supported collaboration scripts. International Journal of Computer Supported Learning, 2(2-3), 211-224.

[33] Tomas Backström, (2004). Collective learning: A way over the ridge to a new organizational attractor, Learning Organization, The, Vol. 11 Iss: 6, pp.466 - 477

[34] Vapnik, V. N., (2000). The Nature of Statistical Learning Theory (2nd Ed.), Springer Verlag.

[35] Wheeler, W. M. (1911). The ant colon as an organism. Journal of Morphology, 22(2), 307-325

[36] Wheeler, 1926, p. 433) [Wheeler, W. M. (1926). Emergent evolution and the social. Science, 64(1662), 433-440

[37] Waldrop, M. Mitchell (1992). Complexity: The Emerging Science at the Edge of Order and Chaos. New York: Simon \& Schuster

[38] Lave, J. (1988). Cognition in Practice: Mind, mathematics and culture in everyday life. New York: Cambridge University Press.

[39] Winograd, T., \& Flores C. F. (1986). Understanding computers and cognition: A new foundation for design. Norwood, NJ.

[40] Dillenbourg P. (1999) What do you mean by collaborative learning? In P. Dillenbourg (Ed) Collaborativelearning: Cognitive and Computational Approaches. (pp.1-19). Oxford: Elsevier

[41] Florida, R. (2002) The Rise of the Creative Class and How It's Transforming Work, Leisure, Community and Everyday Life, Basic Books, New York, NY.

[42] IEEE 1471 Website. Retrieved December 22, 2010 from http://www.iso-architecture.org/ieee$\underline{1471 / \text { index.html }}$

[43] ISO/IEC 42010, ANSI/IEEE Std 1471: Conceptual framework. Retrieved December 22, 2010 from http://www.iso-architecture.org/ieee-1471/conceptual-framework.html

[44] TOGAF 8.1.1 Online. Architecture Patterns. Retrieved December 22, 2010 from http://www.opengroup.org/architecture/togaf8-doc/arch/chap28.html

[45] Vengerov, A. (2009). The Use of Fuzzy Transformational Qualifiers in the Organization of Distributed Adaptive Learning. The International Journal of Learning, Common Ground Publishing Pty Ltd, vol. 16, Melbourne, Australia.

[46] Hannafin, M., Land, S., \& Oliver, K. (1999). Open learning environments: Foundations, methods, and models. In C. Reigeluth (Ed.), Instructional Design Theories and Models (pp. 115-140). Mahwah, NJ: Lawrence Erlbaum Associates

[47] Vengerov A. (2008). Diversity and Adaptive Distributed Learning in New Wave Organizations, Journal of Diversity Management, Vol. 3, Number 2, 2008, pp.29-37

[48] IMS Learning Design specification. Retrieved December 22, 2010 from http://www.imsglobal.org/learningdesign/index.html 
\begin{tabular}{ll} 
International Journal of Management \& Information Systems - First Quarter $2012 \quad$ Volume 16, Number 1 \\
\hline
\end{tabular} NOTES 